\title{
Exploring Beyond the DNA Sequence: A Review of Epigenomic Studies of DNA and Histone Modifications in Dementia
}

\author{
Lachlan F. MacBean $^{1}$ - Adam R. Smith ${ }^{1} \cdot$ Katie Lunnon ${ }^{1}$ \\ Published online: 3 August 2020 \\ (C) The Author(s) 2020
}

\begin{abstract}
Purpose of Review Although genome-scale studies have identified many genetic variants associated with dementia, these do not account for all of disease incidence and so recently attention has turned to studying mechanisms of genome regulation. Epigenetic processes such as modifications to the DNA and histones alter transcriptional activity and have been hypothesized to be involved in the etiology of dementia. Here, we review the growing body of literature on dementia epigenomics, with a focus on novel discoveries, current limitations, and future directions for the field.

Recent Findings It is through advances in genomic technology that large-scale quantification of epigenetic modifications is now possible in dementia. Most of the literature in the field has primarily focussed on exploring DNA modifications, namely DNA methylation, in postmortem brain samples from individuals with Alzheimer's disease. However, recent studies have now begun to explore other epigenetic marks, such as histone modifications, investigating these signatures in both the brain and blood, and in a range of other dementias.

Summary There is still a demand for more epigenomic studies to be conducted in the dementia field, particularly those assessing chromatin dynamics and a broader range of histone modifications. The field faces limitations in sample accessibility with many studies lacking power. Furthermore, the frequent use of heterogeneous bulk tissue containing multiple cell types further hinders data interpretation. Looking to the future, multi-omic studies, integrating many different epigenetic marks, with matched genetic, transcriptomic, and proteomic data, will be vital, particularly when undertaken in isolated cell populations, or ideally at the level of the single cell. Ultimately these studies could identify novel dysfunctional pathways and biomarkers for disease, which could lead to new therapeutic avenues.
\end{abstract}

Keywords Alzheimer's disease · Biomarker · Dementia · DNA methylation · Epigenetics · Histone modifications

\section{Introduction}

Dementia is an umbrella term used to describe a group of specific symptoms, including global deterioration of intellect, cognition, and behavior, which arise in a vast range of neurodegenerative conditions [1]. The biggest risk factor associated with dementia is age, with approximately two in every 100 people developing the syndrome between the ages of 65 and

This article is part of the Topical Collection on Neurogenetics and Psychiatric Genetics

Katie Lunnon

k.lunnon@exeter.ac.uk

1 University of Exeter Medical School, RILD Building Level 4, Royal Devon and Exeter Hospital, Barrack Rd, Exeter EX2 5DW, UK
69 , rising after the age of 85 to one in five [2]. Current estimates indicate that approximately 44 million people are living with dementia worldwide [3]. Furthermore, healthcare advances mean that more people are now living to old age and so this number is expected to triple by 2050 .

Alzheimer's disease (AD) is the most prevalent type of dementia, accounting for approximately 50-70\% of all cases [2]. Clinically $\mathrm{AD}$ is characterized by memory loss and mood and personality changes. Neuropathologically the disease is characterized by the accumulation of extracellular amyloid beta $(\mathrm{A} \beta)$ plaques, intracellular neurofibrillary tangles (NFTs), and neuroinflammation. A $\beta$ plaques are produced from the incorrect cleavage of amyloid precursor protein (APP) by $\beta$ - and $\gamma$-secretases, while NFTs are paired helical filaments of hyperphosphorylated tau protein. The characteristic spread of both these pathological hallmarks has been used to develop postmortem staging criteria 
[4-6]. Aside from AD, there are several other neurodegenerative diseases characterized by dementia that burden the elderly (Table 1). Vascular dementia (VaD) is the second most prevalent dementia, accounting for $\sim 20 \%$ of dementia cases [7], and commonly arises because of brain infarcts, resulting from a blood supply loss. Conversely, dementia with Lewy bodies (DLB) accounts for $10-15 \%$ of dementia cases and is characterized by the cytoplasmic accumulation of $\alpha$-synuclein in neurons, forming Lewy bodies (LBs). Pathologically, DLB is difficult to discriminate postmortem from Parkinson's disease dementia (PDD) and these diseases are only distinguished clinically by the arbitrary timing of motor symptoms relative to cognitive changes [13]. Other less common neurodegenerative diseases that feature dementia include frontotemporal dementia (FTD) and Huntington's disease (HD). Although various disorders that feature dementia can be neuropathologically classified into distinct conditions, it is worth noting that mixed dementia is common, for example many individuals with $\mathrm{AD}$ pathology will also have some co-existing Lewy body pathology $[14,15]$.

\section{The Contribution of Genetic Variation to Dementia Etiology}

$\mathrm{AD}$ can be divided in to two subtypes: familial $\mathrm{AD}(\mathrm{FAD})$ and sporadic AD (SAD). Sources vary in their estimation of FAD

Table 1 Characteristics of the major neurodegenerative disorders characterized by dementia

\begin{tabular}{|c|c|c|c|}
\hline Condition & $\begin{array}{l}\text { Approximate prevalence } \\
\text { across all dementia cases }\end{array}$ & Key pathology & Clinical characteristics \\
\hline Alzheimer's disease (AD) & $50-70 \%$ of dementia cases [3] & $\begin{array}{l}\text { Build-up of amyloid beta plaques and } \\
\text { neurofibrillary tangles (NFTs) initially within } \\
\text { the trans-entorhinal cortex. } \\
\text { Eventually pathology spreads to the } \\
\text { hippocampus and neocortex. }\end{array}$ & $\begin{array}{l}\text { Memory impairment, confusion, poor judgment, } \\
\text { language disturbance, visual complaints, } \\
\text { agitation, withdrawal, and hallucinations. }\end{array}$ \\
\hline Vascular dementia (VaD) & $\sim 17 \%$ of dementia cases [7] & $\begin{array}{l}\text { Infarcts resulting from a failure of blood supply } \\
\text { to the affected brain region through, for } \\
\text { example, a stroke. }\end{array}$ & $\begin{array}{l}\text { Memory impairment, confusion, depression, } \\
\text { apathy, and agitation more prevalent } \\
\text { compared with AD. }\end{array}$ \\
\hline $\begin{array}{l}\text { Dementia with Lewy } \\
\text { bodies (DLB) }\end{array}$ & $4.6 \%$ of dementia cases [8] & $\begin{array}{l}\text { Lewy-type pathology: build-up of } \\
\text { alpha-synuclein deposits in nerve cells in the } \\
\text { cerebral and limbic cortex, hippocampus, and } \\
\text { basal ganglia. } \\
\text { Severity correlates with the abundance of Lewy } \\
\text { bodies in the cortex. }\end{array}$ & $\begin{array}{l}\text { Memory impairment, confusion, fluctuating } \\
\text { cognition, parkinsonism, visual } \\
\text { hallucinations, REM sleep behavioral } \\
\text { disorder, general cognitive decline, and } \\
\text { memory impairment. }\end{array}$ \\
\hline $\begin{array}{l}\text { Frontotemporal dementia } \\
\text { (FTD) }\end{array}$ & $\sim 3 \%$ of dementia cases [9] & $\begin{array}{l}\text { Deposits of hyperphosphorylated tau, } \\
\text { abnormally folded and hyperphosphorylated } \\
\text { TDP-43, or fused sarcoma protein in the } \\
\text { frontotemporal region leading to lobar } \\
\text { atrophy. }\end{array}$ & $\begin{array}{l}\text { Three clinical variants: behavioral-variant FTD } \\
\text { including personality changes and apathy; } \\
\text { non-fluent variant primary progressive } \\
\text { aphasia (nfvPPA); and semantic-variant } \\
\text { primary progressive aphasia (svPPA). } \\
\text { Progression leads to convergence of the } \\
\text { clinical variants. Global cognitive } \\
\text { impairment, parkinsonism, and motor neuron } \\
\text { disease are often observed. } \\
\text { Memory impairment and confusion are more } \\
\text { common in advanced cases. }\end{array}$ \\
\hline $\begin{array}{l}\text { Parkinson's disease } \\
\text { dementia } \\
\text { (PDD) }\end{array}$ & $\begin{array}{l}3.6 \% \text { of dementia cases }[10] \\
\text { (Note: Prevalence of PD } \\
\text { patients exhibiting } \\
\text { dementia-like symptoms is } \\
24.5 \% \text { ) [8] }\end{array}$ & $\begin{array}{l}\text { Alpha-synuclein deposits spreading from the } \\
\text { nervous system to the cortical areas may be } \\
\text { associated with the development of dementia } \\
\text { in PD. } \\
\text { Pathologically similar to DLB. }\end{array}$ & $\begin{array}{l}\text { Dementia, on average, emerges around } 18 \text { years } \\
\text { after PD duration. } \\
\text { Common symptoms of PD include tremor, } \\
\text { bradykinesia, bradyphrenia, pain, fatigue, } \\
\text { sleepiness, and mood disorders. } \\
\text { Executive decline, slowed cognition, short-term } \\
\text { memory impairment, and confusion are well } \\
\text { reported in PDD. }\end{array}$ \\
\hline Huntington's disease (HD) & $\begin{array}{l}\text { 10.6-13.7 individuals per } \\
100,000 \text { in western } \\
\text { populations }[11,12] \\
\text { (Note: Prevalence of } \\
\text { Huntington's disease } \\
\text { dementia } \\
\text { highly inconsistent between } \\
\text { studies, dependent on } \\
\text { dementia criteria) }\end{array}$ & $\begin{array}{l}\text { The appearance of nuclear and cytoplasmic } \\
\text { inclusions containing mutant huntingtin and } \\
\text { polyglutamine in the caudate and putamen } \\
\text { regions of the striatum. }\end{array}$ & $\begin{array}{l}\text { Memory loss is common but should be coupled } \\
\text { with other cognition criteria to avoid } \\
\text { inaccurate diagnosis. Speed of processing is } \\
\text { highly impaired in HD dementia, as is } \\
\text { attention span, executive functions, and } \\
\text { visuospatial abilities. }\end{array}$ \\
\hline
\end{tabular}

Approximate prevalence is an estimate and does not represent a completely accurate proportion across the entire population of dementia patients. Prevalence, pathology, and clinical characteristics have been comprehensively reviewed in several publications [3, 7, 9-13] 
prevalence, but overall predict that FAD is responsible for approximately $1-10 \%$ of all AD cases with symptoms usually observed before the age of 65 [16-18]. FAD is caused by one or more autosomal dominant mutations in the $A P P$ gene or in the presenilin $(P S E N) 1$ and 2 genes, which encode components of the $\gamma$-secretase. Contrastingly, SAD tends to be lateonset (LOAD) and is thought to result from the interplay between multiple genetic and environmental factors. Owing to large-scale meta-analyses of genome-wide association studies (GWAS) in recent years, a number of single nucleotide polymorphisms (SNPs) have been implicated in the development of $\mathrm{SAD} / \mathrm{LOAD}$, featuring genes in specific pathways such as lipid metabolism, APP processing, and inflammation [19-21]. Although alone these common variants only contribute a relatively small amount to disease risk, recent studies have combined these together to generate polygenic risk scores (PRS), which can even be used to predict the likelihood of an individual developing AD with $\sim 78 \%$ accuracy [22]. Comparatively, there are far fewer well-powered genomic studies in other common dementias to date. For example, the first large-scale GWAS in DLB was only published in 2018, which identified risk loci that are shared with both $\mathrm{AD}$ (e.g., the $A P O E$ \& 4 allele) and Parkinson's disease (PD) (e.g., $S N C A)$ as well as some novel loci that may be specific to DLB [23, 24].

Although GWAS have provided valuable insight into the genetic variants associated with the development of dementia, particularly in the context of AD, these SNPs do not account for all of disease incidence. As such, attention in recent years has turned to studying mechanisms of regulating the genome, such as epigenetic processes.

\section{Contextualizing the Epigenome}

Epigenetics refers to the reversible regulation of gene expression, occurring independent of DNA sequence variation through modifications to the DNA or histone proteins or non-coding RNAs (ncRNAs) (Fig. 1). These epigenetic mechanisms can be dynamic, changing in response to environmental stimuli. In the context of complex disease phenotypes, research in recent years has focussed on characterizing epigenetic profiles, with the aim of discovering novel therapeutic targets and biomarkers.

The best characterized epigenetic modification is that of DNA methylation, whereby a methyl group is added to the C5 position of a cytosine residue forming 5-methylcytosine $(5-\mathrm{mC})$ [25]. While DNA methylation was traditionally viewed to result in gene silencing, recent evidence suggests that this is dependent on the genomic context, and in fact 5$\mathrm{mC}$ can be associated with increased gene expression or alternative splicing [26]. Aside from 5-mC, several other DNA modifications have been described, including 5- hydroxymethylcytosine (5-hmC), 5-carboxylcytosine (5$\mathrm{caC})$, and 5 -formylcytosine (5-fC) (Table 2). These were initially thought to be intermediates in the demethylation pathway back to an unmodified cytosine; however, recent evidence suggests that they may have functional roles in their own right [27]. Indeed, 5-hmC has been shown to be present at relatively high levels in the brain compared with other tissues, particularly in synaptic genes [28]. Aside from direct modifications to the DNA, the genome can also be regulated by post-translational modification of histone proteins (e.g., acetylation, ubiquitination, methylation, phosphorylation, and SUMOylation), which contribute to the regulation of chromatin accessibility, and thus transcriptional suppression and activation [29] (Table 2). Finally, gene expression can also be regulated by ncRNAs, which are short RNA strands transcribed from the DNA sequence that are not subsequently translated. A multitude of ncRNA species exist within the human genome, each tailored to a specific role, and these have been previously reviewed extensively in the context of dementia [30] and thus will not be a focus of this review.

\section{Profiling DNA Modifications in Postmortem Dementia Brain Samples}

Many of the initial epigenetic studies in dementia focussed on profiling DNA methylation in specific candidate genes, for example those implicated in $A \beta$ or tau generation (see Roubroeks et al. [31] for a comprehensive review). However, such studies were often inconclusive, owing to differences between tissue type, samples, and techniques used [32-36]. Other early studies focussed on exploring global DNA modification patterns in postmortem brain tissue using techniques such as immunocytochemistry with antibodies specific to different DNA modifications [37-40]. However, this approach can only detect average changes in DNA modification across the whole genome and cannot identify alterations at single nucleotide resolution. In addition, many of these studies were also inconclusive due to similar limitations that were observed in the candidate gene studies (as reviewed in Roubroeks et al. [31]). More recently, advances in genomic technology have allowed large-scale quantification of DNA methylation at single nucleotide resolution in dementia via epigenome-wide association studies (EWAS) (Table 3).

The first EWAS in dementia was published in 2012, utilizing the Illumina Infinium Human Methylation $27 \mathrm{~K}$ BeadChip array ( $27 \mathrm{~K}$ array) to profile $\sim 27,000$ loci and identified differential methylation across $948 \mathrm{CpG}$ sites, representing 918 genes, in human frontal cortex tissue from $12 \mathrm{AD}$ and 12 nondemented control donors [57]. In 2014, two EWAS of AD were published back-to-back, which utilized the Illumina Infinium Human Methylation $450 \mathrm{~K}$ BeadChip array (450 K array) to investigate DNA methylation patterns at $\sim 450,000$ 
Fig. 1 DNA is tightly wound around histone proteins for compaction into chromatin, and subsequently chromosomes, and gene regulation. (A) The binding of epigenetic factors to histone tails can alter the availability of the genes in the DNA for activation. (B) Direct DNA modifications, such as the addition of a methyl group to a cytosine residue, may also change the activity of a DNA region without changing the sequence itself. (C) microRNAs also function in post-transcriptional regulation of gene expression via complementary base pairing

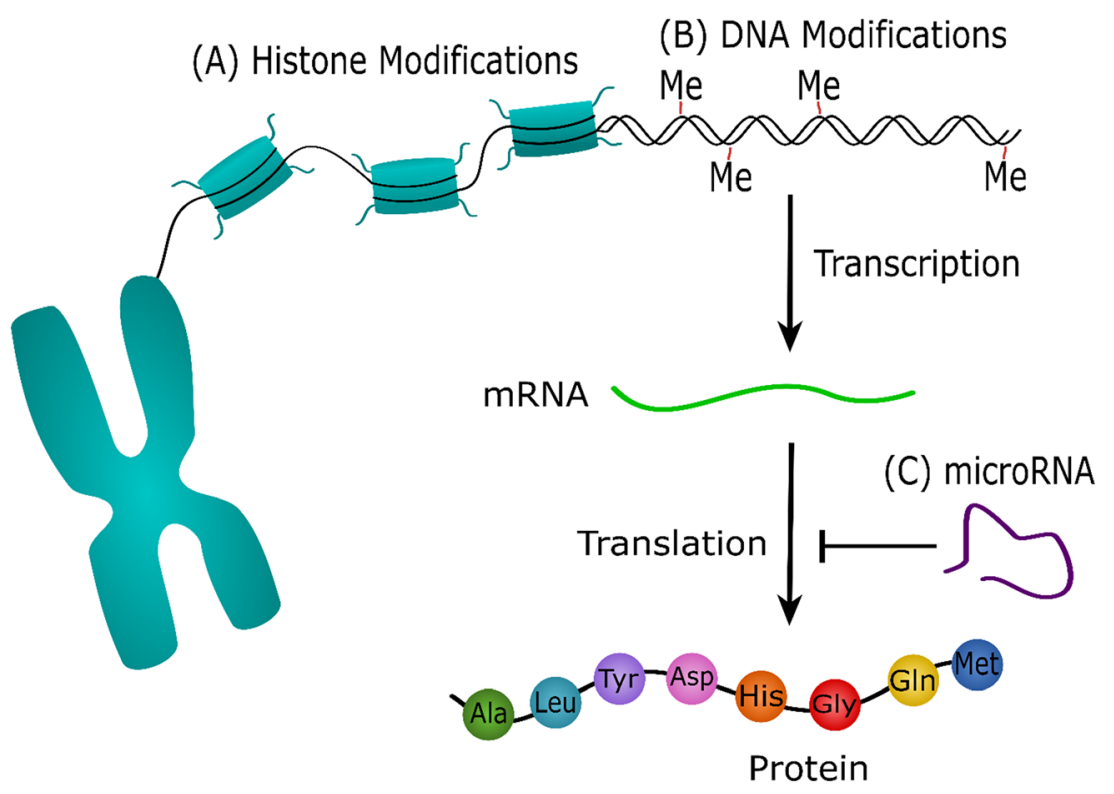

loci in far larger cohorts. De Jager and colleagues profiled prefrontal cortex brain tissue from $>700$ donors with varying degrees of $\mathrm{AD}$ pathology to identify 71 differentially methylated loci, of which 11 were validated in the accompanying article by Lunnon and colleagues [53••]. The study by Lunnon et al. performed a cross-cortex brain EWAS utilizing matched $450 \mathrm{~K}$ array data from three cortical brain regions (entorhinal cortex, superior temporal gyrus, prefrontal cortex) and the cerebellum in $\sim 120$ individuals $[55 \bullet \bullet]$. They reported significant cortical hypermethylation of the ANK1 gene with increasing Braak stage (used as a measure of NFT spread), which they replicated in three further cortical datasets in addition to showing distinct DNA methylation patterns in $\mathrm{AD}$ in the brain and the blood. More recently, a number of other teams have utilized the $450 \mathrm{~K}$ array to study DNA methylomic variation in $\mathrm{AD}$; Watson et al. discerned 479 differentially methylated regions (DMRs) which exhibited "strong bias for hypermethylated changes" in 34 LOAD and 34 control superior temporal gyrus samples, overlapping with genes previously reported to be involved in neuron function, development, and cellular metabolism [52]. Similarly, Smith and colleagues analyzed $450 \mathrm{~K}$ array data from 147 individuals in the superior temporal gyrus and identified most notably a DMR spanning $48 \mathrm{~kb}$ across the HOXA gene cluster, which was hypermethylated with increasing Braak stage [48]. More recently, Altuna et al. utilized the $450 \mathrm{~K}$ array to quantify DNA methylation in the hippocampus of $26 \mathrm{AD}$ and 12 control brain samples, reporting $118 \mathrm{AD}$ differentially methylated positions (DMPs), including HOXA3, a previously reported gene of interest. These loci were enriched in genes associated with brain and embryonic morphogenesis and homeoboxcontaining transcription factors [42].

The brain is a heterogeneous tissue and it is well reported that there are alterations in the abundance and activity of various cell types in AD. One key limitation of the aforementioned studies is that they have been undertaken on bulk brain tissue. Although many of these have attempted to control for changes in cell proportions bioinformatically, this does not allow the exploration of cell-type specific DNA methylation changes. As such, a couple of recent studies have focussed on profiling DNA methylation in isolated cell types in AD brain. Gasparoni et al. profiled bulk tissue DNA methylation patterns in 63 frontal and 65 temporal cortex samples derived from 52 healthy and $76 \mathrm{AD}$ donors using the $450 \mathrm{~K}$ array. They also profiled DNA methylation in sorted neuronal and non-neuronal (glia) nuclei isolated from 31 occipital cortex samples, to identify cell-type specific DNA methylation patterns [46]. Using this approach, the authors concluded that DNA methylation changes in $H O X A 3$ were driven by alterations in neuronal cells, while changes in ANK1 were driven by alterations in glial cells. When the authors meta-analyzed the neuronal and glial analyses, they identified a number of enriched pathways of functional relevance, for example KEGG pathways for Alzheimer's disease, neurotrophin signaling, and MAPK signaling. One other study has investigated cell-type specific DNA methylation changes in AD; Mano and colleagues profiled DNA methylation in neurons isolated from the inferior temporal gyrus in $30 \mathrm{AD}$ patients and 30 agematched normal controls, identifying eight DMRs and highlighting the BRCAl gene, which also showed protein changes in disease [65].

Although the focus of most DNA methylomic studies in dementia to date has been in the context of $\mathrm{AD}$, some other diseases have been investigated, albeit with far fewer samples. The first of two studies that have interrogated DNA methylation status in DLB used the GoldenGate DNA Methylation BeadArray to compare DNA methylation patterns across a large number of different tissue types and disease phenotypes, 
Table 2 Epigenetic processes that are involved in gene regulation

\begin{tabular}{|c|c|c|}
\hline Modification & Definition & Functional consequence \\
\hline \multicolumn{3}{|c|}{ Cytosine modifications } \\
\hline DNA 5-methylcytosine (5-mC) & $\begin{array}{l}\text { The methylated form of cytosine. } \\
\text { A methyl group is attached to the } 5 \text { th cytosine of the pyrimidine } \\
\text { ring via DNA methyltransferases (DNMTs). }\end{array}$ & $\begin{array}{l}\text { The addition of a methyl group to a cytosine residue } \\
\text { primarily acts by recruiting proteins involved in gene } \\
\text { repression. }\end{array}$ \\
\hline $\begin{array}{l}\text { DNA 5-hydroxymethylcytosine } \\
\text { (5-hmC) }\end{array}$ & $\begin{array}{l}\text { The first oxidative product in the demethylation of 5-mC. } \\
5 \text {-mC is oxidized to 5-hmC via ten-eleven translocation (TET) } \\
\text { enzymes. }\end{array}$ & $\begin{array}{l}\text { It is thought that } 5-\mathrm{hmC} \text { interacts with chromatin-binding } \\
\text { proteins distinct from those recruited by } 5-\mathrm{mC} \text { to } \\
\text { regulate gene expression. }\end{array}$ \\
\hline DNA 5-formylcytosine (5-fC) & $\begin{array}{l}\text { The second oxidative product in the demethylation pathway. } \\
5 \text {-hmC is oxidized to } 5 \text {-fC in a further step by TET. }\end{array}$ & DNA demethylation intermediate. \\
\hline DNA 5-carboxylcytosine (5-caC) & $\begin{array}{l}\text { The third and final oxidative product in the demethylation } \\
\text { pathway. } \\
\text { The oxidation of 5-fC to 5-caC is catalyzed by TET. } \\
\text { 5-caC can then be converted to an unmodified cytosine via } \\
\text { thymine DNA glycosylase (TDG). }\end{array}$ & DNA demethylation intermediate. \\
\hline Histone acetylation & $\begin{array}{l}\text { Histone modifications } \\
\text { As part of gene regulation, an acetyl moiety is introduced to lysine } \\
\text { residues within the N-terminal tail of the histone core. }\end{array}$ & $\begin{array}{l}\text { Typically, the negative charge added by acetylation results } \\
\text { in relaxation of the chromatin structure. This in turn } \\
\text { allows transcription factors to bind the open sequence } \\
\text { increasing expression. }\end{array}$ \\
\hline Histone methylation & $\begin{array}{l}\text { A process involving the addition of a methyl group to the amino } \\
\text { acids of histone proteins. }\end{array}$ & $\begin{array}{l}\text { Methylation of arginine residues may promote } \\
\text { transcription, while lysine methylation is implicated in } \\
\text { both activation and repression of transcription. }\end{array}$ \\
\hline Histone phosphorylation & $\begin{array}{l}\text { Often activated in response to DNA damage or as an intermediate } \\
\text { step in chromosome condensation, phosphorylation is a } \\
\text { post-translational modification involving the addition of a } \\
\text { phosphate group to core histone proteins. }\end{array}$ & $\begin{array}{l}\text { Histone phosphorylation acts as a platform for } \\
\text { chromatin-associated factors and effector proteins and } \\
\text { is associated with active gene transcription. }\end{array}$ \\
\hline Histone ubiquitination & $\begin{array}{l}\text { The attachment of a ubiquitin protein to a core histone protein } \\
\text { plays a central role in the DNA damage response. This can be } \\
\text { mono (single ubiquitin protein) or poly (a chain of ubiquitin). }\end{array}$ & $\begin{array}{l}\text { Ubiquitination of specific histones has varying effects on } \\
\text { gene regulation, for example, monoubiquitinating of } \\
\mathrm{H} 2 \mathrm{~A} \text { is associated with gene silencing while H2B is } \\
\text { associated with transcription activation. }\end{array}$ \\
\hline
\end{tabular}

including the cerebral cortex from 13 DLB patients [62]. Although the authors did highlight that the pattern of hypomethylation in the DLB samples distinguished these donors from normal brain and neuroectodermal tumors (glioma and neuroblastoma), it was not clear whether the same brain region was used for these comparisons. In the other study, SanchezMut and colleagues used the $450 \mathrm{~K}$ array and subsequent pyrosequencing to profile DNA methylation in the gray matter in the dorsolateral prefrontal cortex in a small cohort of $\mathrm{AD}$, DLB, PD, and down syndrome cases with AD (DS-AD) [59]. The authors identified a number of DMRs associated with all the neurodegenerative diseases, highlighting that these were significantly overrepresented in signaling pathways related to brain function (e.g., hippocampus, Wnt, and neurotrophin signaling) and immune function (e.g., TGF- $\beta$ signaling, Fc $\gamma$ Rmediated phagocytosis). The authors also demonstrated differential DNA methylation in ANK1 and RHBDF2 in AD and DLB, corroborating several previous AD EWAS. However, a recent bisulfite pyrosequencing study of ANK1 in different neurodegenerative diseases reported that disease-associated hypermethylation in the entorhinal cortex in DLB donors was only observed when there was co-existing AD pathology [45]. In the context of other neurodegenerative diseases, another study utilizing the $450 \mathrm{~K}$ array demonstrated DNA methylomic alterations in HLA-DQA1, GFPT2, MAPT, and MIR886 in five PD frontal cortex and blood samples compared with six age-matched healthy controls, even though the conclusions that can be drawn from the study are limited by its power [61].

Although the focus of all these studies has been on quantifying DNA methylation patterns in disease, one limitation is that they have used bisulfite-treated DNA and thus have quantified both 5-mC and 5-hmC. However, several recent studies have aimed to profile these modifications in isolation. The first, published by Zhao et al., used a selective chemical labeling technique prior to sequencing to quantify $5-\mathrm{hmC}$ in prefrontal cortex samples from $20 \mathrm{AD}$ and 10 control donors. The authors identified 517 differentially hydroxymethylated regions (DhMRs) significantly associated with neuritic plaques and 60 DhMRs associated with NFTs, although the low sequencing resolution meant it was not possible to discriminate between 5-mC and 5-hmC. Interestingly, of the plaqueassociated DhMRs, these were annotated to genes enriched for neuronal functions, such as long-term memory and neurotrophin signaling [50•]. Later that year, Ellison and colleagues published a study utilizing reduced representation hydroxymethylation profiling (RRHP) to analyze 5-hmC levels in the hippocampus in three $\mathrm{AD}$ and two control donors. 


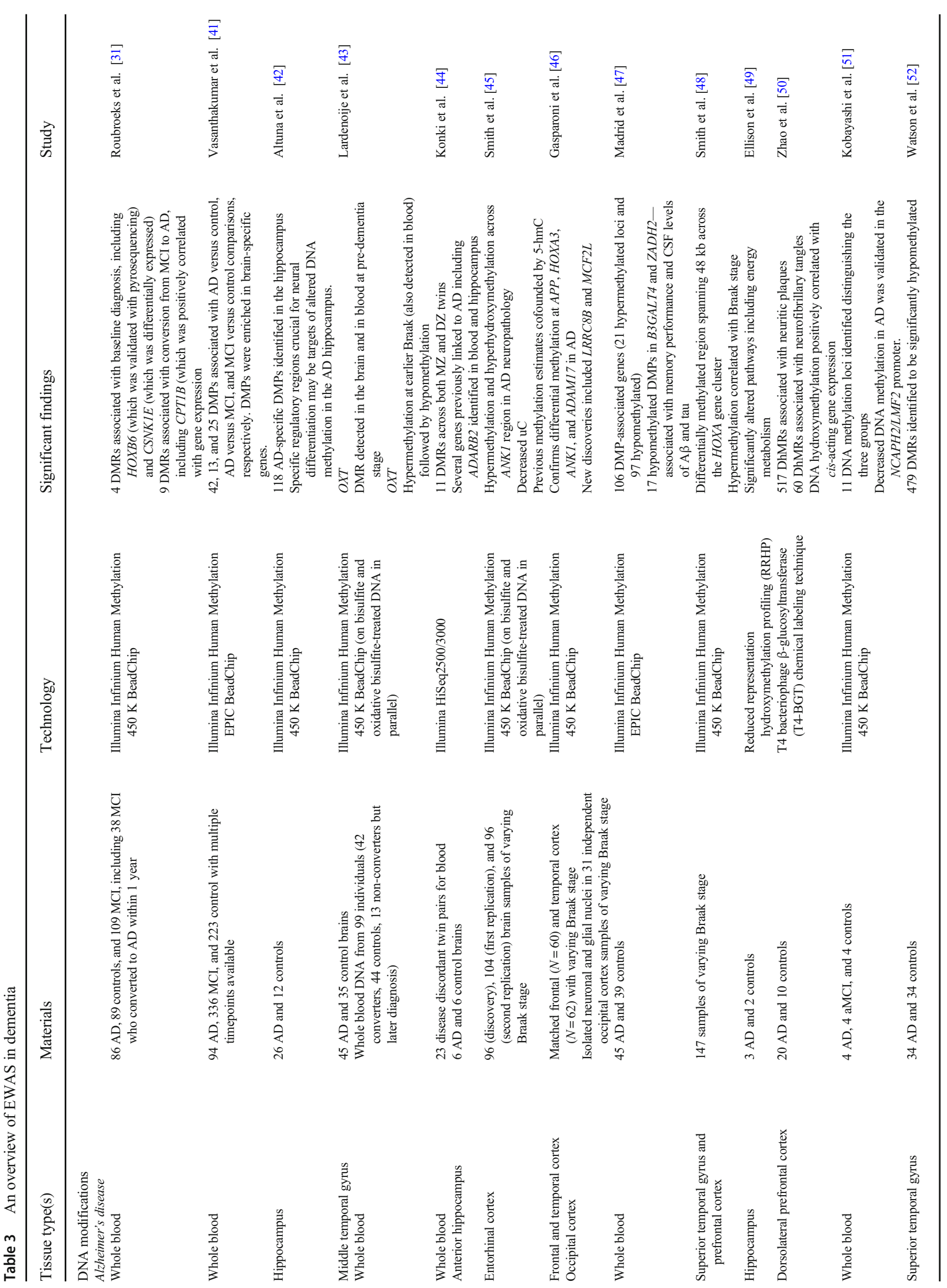




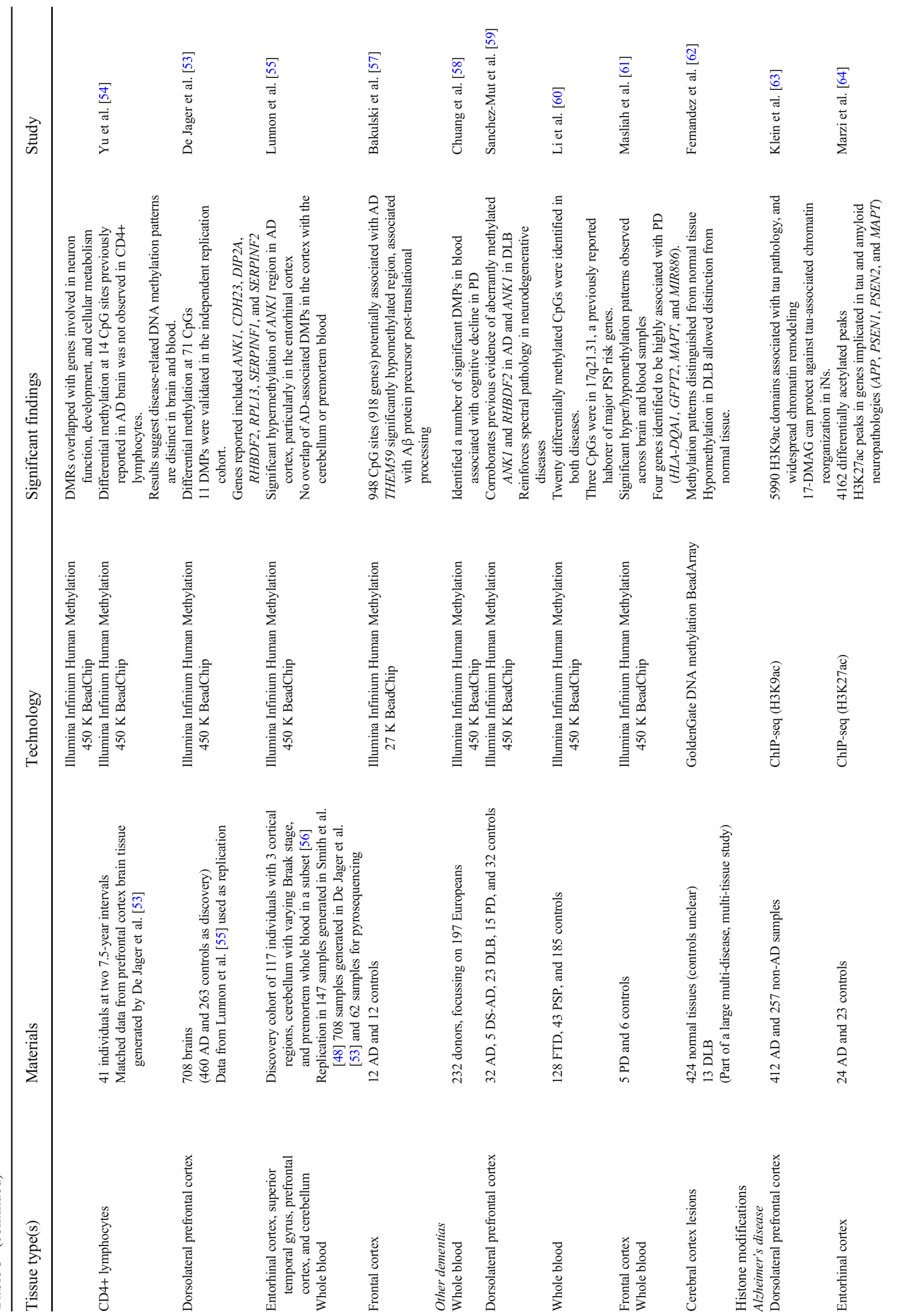


Although the study highlighted several significantly altered pathways including, for example, energy metabolism, further replication is needed in a larger sample cohort. More recently, two studies have used the $450 \mathrm{~K}$ array to quantify 5 -hmC and 5 -mC independently in $\mathrm{AD}$ by oxidizing the DNA prior to bisulfite treatment [49]. Smith et al. used the array to evaluate 5-hmC, 5-mC, and unmodified cytosine $(\mathrm{uC})$ in the entorhinal cortex in 96 individuals with varying degrees of pathology before performing targeted pyrosequencing in a smaller independent replication cohort [66]. They demonstrated that previous estimates of $\mathrm{AD}$-associated hypermethylation in $A N K 1$ were underestimates due to confounding by hypohydroxymethylation, suggesting a loss of active DNA demethylation in AD. More recently, Lardenoije and colleagues used the same approach to quantify 5-hmC, 5-mC, and $\mathrm{uC}$ in the middle temporal gyrus in $45 \mathrm{AD}$ and 35 control donors. They identified a DMR in the OXT gene consisting of ten adjacent $\mathrm{CpG}$ sites, which was hypomethylated in $\mathrm{AD}$ in the temporal cortex and which was also differentially methylated in blood samples from non-demented individuals who subsequently converted to $\mathrm{AD}$ dementia [43].

\section{Understanding Chromatin Dynamics in Postmortem Dementia Brain Samples}

Although the focus of most empirical dementia EWAS has been on profiling DNA modifications, recently, studies have started to investigate histone modifications and chromatin dynamics. To date, two EWAS of histone acetylation have been undertaken in AD brain samples using chromatin immunoprecipitation sequencing (ChIP-seq), which were both published within the last two years. The first profiled histone 3 lysine 27 acetylation (H3K27ac) in the entorhinal cortex of $24 \mathrm{AD}$ and 23 control postmortem brain samples [64••]. They identified 4162 differentially acetylated peaks, including regions annotated to genes implicated in AD pathology, for example APP, PSEN1, PSEN2, and MAPT. Interestingly, the authors also showed a highly significant enrichment of AD risk variants in the H3K27ac peak regions. The authors reported that ADassociated differentially acetylated peaks were enriched in processes related to pathology (e.g., lipoprotein binding, $A \beta$ metabolic process) and neuronal activity (e.g., GABAreceptor activity, synaptic proteins).

Klein and colleagues more recently profiled $\mathrm{H} 3 \mathrm{~K} 9 \mathrm{ac}$ in the dorsolateral prefrontal cortex in a larger cohort size of 669 donors, 412 of whom were AD patients [63*0]. They identified 26,384 H3K9ac peaks (domains), of which $23 \%$ (5990) showed an association with tau pathology and $2 \%$ (602) were associated with amyloid pathology, with only 88 domains significantly associated with both pathologies. The authors showed that the tau-related changes accumulated in large genomic segments, indicating spatial chromatin organization, while there were no domains co-ordinately enriched for associations with amyloid pathology. Interestingly, the study also suggested a potential therapeutic for altering the widespread chromatin remodeling associated with tau pathology. The authors identified compounds within the Connectivity Map (CMAP) database whose gene expression signature was negatively correlated with the tau pathology-associated H3K9ac signature and reported that the N-terminal Heat Shock Protein 90 (Hsp90) inhibitor 17-(dimethylaminoethylamino)-17demethoxygeldanamycin (17-DMAG, alvespimycin) was the most significant compound. When they later treated induced neurons (iNs) with this compound, they demonstrated this protected against tau-associated chromatin reorganization, highlighting the translational potential of EWAS for identifying new therapeutics.

\section{Identifying Epigenomic Biomarkers in Blood}

As epigenomes are cell-type specific, most empirical EWAS in dementia to date have therefore been undertaken in various regions of the cortex. However, as human samples are only available postmortem, this approach allows only a "snapshot," often at the end stage of disease. There is growing interest in profiling epigenetic processes in blood samples from individuals with dementia in the hope of identifying disease-specific signatures. There are a number of different epigenetic biomarkers that could have clinical utility; diagnostic biomarkers could be used to distinguish between individuals with different types of dementia, while predictive biomarkers for future cognitive decline could be used to monitor the response of individuals to new therapeutics. A recent systematic review of DNA methylation studies in AD blood samples highlighted 48 studies that have quantified DNA methylation in peripheral blood samples with respect to dementia status [67]. The majority of the studies they identified analyzed global DNA methylation or the methylation status of specific candidate genes. The authors did highlight some EWAS, which are discussed individually below along with some more recent studies, although many of these are too underpowered to draw substantial conclusions.

Lunnon and colleagues presented DNA methylation profiles in whole blood collected from a subset $(N=57)$ of the donors from their cross-cortex EWAS using the $450 \mathrm{~K}$ array. While they identified several DMPs in blood associated with $\mathrm{AD}$, none were significant when accounting for multiple testing, and there was negligible overlap with cortex or cerebellum methylation profiles [55••]. Kobayashi et al. also used the $450 \mathrm{~K}$ array to analyze DNA methylation in four $\mathrm{AD}$, four amnestic mild cognitive impairment (aMCI), and four nondemented control whole blood samples [51]. The authors then used pyrosequencing to validate decreased DNA methylation in the NCAPH2/LMF2 promoter region in additional blood samples. Another study used the $450 \mathrm{~K}$ array to profile 
DNA methylation in premortem CD4+ lymphocytes collected from 41 individuals at two timepoints, approximately 7.5 years apart, alongside matched prefrontal cortex brain tissue collected at death approximately a year after the second blood sample [54]. The authors then explored whether there was any association of AD with DNA methylation in CD4+ lymphocytes at $14 \mathrm{CpG}$ sites they had previously shown to be differentially methylated with respect to neuritic plaque burden in the prefrontal cortex [53••]. However, they observed no differential methylation at these loci in CD4+ lymphocytes, again suggesting that DNA methylation profiles in disease are distinct in the brain and blood [54, 55]. The systematic review also highlighted one EWAS that used the $450 \mathrm{~K}$ array to quantify DNA methylation in peripheral blood in tauopathies, comparing 128 FTD, 43 progressive supranuclear palsy (PSP), and 185 control individuals [60]. The authors identified $20 \mathrm{CpGs}$ being differentially methylated in both diseases compared with controls, including three $\mathrm{CpGs}$ in $17 q 21.31$, which has been previously shown to contain the major genetic risk factor for PSP. Gene ontology analysis highlighted an overrepresentation of differentially methylated loci in PSP in genes annotated to pathways such as DNA or transcription factor binding [56].

Since the systematic review from Fransquet and colleagues [67], a handful of further EWAS in dementia blood samples have been published. The two most recent studies have both used large cross-center cohorts, yielding the most comprehensive studies to date. Most recently, Roubroeks and colleagues used the $450 \mathrm{~K}$ array to quantify DNA methylation in $86 \mathrm{AD}$, 89 controls, and 109 MCI subjects, including $38 \mathrm{MCI}$ subjects who converted to AD within one year of blood sampling [31]. They identified four significant DMRs associated with baseline diagnosis and nine significant DMRs associated with MCI conversion to AD, validating their most significant DMR in the baseline analysis (residing in $H O X B$ ), using pyrosequencing. Interestingly, several of the DMRs resided in genes that showed transcriptional changes in the same samples; the CSNK1E gene which showed hypermethylation in MCI also showed significantly increased gene expression in the same MCI and AD subjects relative to controls, although there was no correlation between methylation and expression. Similarly, there was a significant correlation of methylation and transcription for the CPT1B gene, which was identified in the MCI conversion analysis. Weighted gene correlation network analysis (WGCNA) identified modules (clusters) of comethylated loci associated with key variables such as diagnosis, neuroimaging measures, and $A P O E$ genotype, with downstream pathway analyses on these genes highlighting immune processes. Vasanthakumar and colleagues recently used the Illumina Infinium Human Methylation EPIC BeadChip array (EPIC array), which has nearly twice the coverage of its predecessor, to profile DNA methylation in whole blood samples collected as part of the Alzheimer's disease neuroimaging initiative (ADNI) consortium [41]. They quantified DNA methylation in 653 individuals (94 AD, 336 MCI, 223 control) across multiple timepoints, identifying between the AD versus control, AD versus MCI, and MCI versus control groups 42, 13, and 25 DMPs at a relaxed $p$ value threshold of $p<1 \times 10^{-5}$, respectively, which were enriched in brainspecific genes. Lardenoije and colleagues used the $450 \mathrm{~K}$ array to profile DNA methylation in whole blood samples collected before and after clinical conversion to $\mathrm{AD}$, including 54 individuals who later developed dementia (AD converters) and 42 controls [43]. Their analyses focussed on a DMR in the $O X T$ gene, which was hypermethylated in converters. Interestingly, this region had been significantly hypomethylated in brain tissue they had also profiled in an independent set of donors, highlighting a potential premortem predictive biomarker for the onset of AD. Madrid and colleagues recently profiled DNA methylation in 45 LOAD and 39 control whole blood samples using the EPIC array [47]. The authors identified 477 disease-associated DMPs when comparing LOAD versus non-LOAD, of which 17 overlapped with DMPs identified using six other continuous variables in the same tissue, including Rey Auditory Verbal Learning Test scores, measurements of cerebrospinal fluid (CSF) tau and phosphorylated tau, and $A \beta_{40}$ and $A \beta_{42}$ ratios. These 17 DMPs were all hypomethylated in LOAD, with 12 residing in $B 3 G A L T 4$ and five located in $Z A D H 2$ gene regions.

Another recent study focussed on profiling peripheral blood DNA methylation in 23 Finnish twin pairs discordant for $\mathrm{AD}$ using reduced representation bisulfite sequencing (RRBS) [44]. They identified 11 significant genomic regions with $>15 \%$ median DNA methylation differences between twin pairs. The authors focussed their validation on the $A D A R B 2$ DMR, as they also showed this was differentially methylated in AD anterior hippocampus in a small cohort of 12 donors. The authors subsequently used bisulfite pyrosequencing to validate $\mathrm{AD}$-associated hypermethylation in the $A D A R B 2$ DMR in blood in a separate cohort of 62 discordant twin pairs. Interestingly, when they then profiled 120 twin pairs prior to disease onset, they observed no difference, suggesting that this gene is not predictive for developing $\mathrm{AD}$ and only becomes hypermethylated after the onset of disease.

Chuang and colleagues used the $450 \mathrm{~K}$ array to profile peripheral whole blood samples collected from 232 PD patients with longitudinal cognitive follow-up, focussing their analyses on 197 individuals of European ancestry [58]. They identified seven genome-wide significant $\mathrm{CpGs}$ associated with cognitive decline, suggesting that it may be possible to identify epigenetic signatures associated with the development of PDD. WGCNA identified modules of comethylated genes, with those associated with motor progression enriched for genes involved in mitochondrial apoptosis, synaptic functions, and Wnt signaling. 
Although existing EWAS in dementia blood samples have largely been undertaken using peripheral whole blood samples, there is speculation that cell-free DNA (cfDNA) circulating in plasma could serve as a novel source of biomarker for neurodegenerative disease, representing DNA fragments that originated from dying neurons or other brain cells. cfDNA methylation patterns can reveal their cellular origin and such signatures have been studied in the context of cancer detection [68]. There is current interest regarding the potential for cfDNA as a biomarker candidate for neurodegenerative disease; indeed, a recent study of cfDNA isolated from 20 ALS and 20 control individuals highlighted a novel differentially methylated mark in the RHBDF2 gene in ALS patients [69]. Interestingly, this gene has been previously shown to be differentially methylated in $\mathrm{AD}$ cortex in a number of EWAS $[53,55,70]$. Therefore, profiling this locus longitudinally in plasma cfDNA in pre-clinical $\mathrm{AD}$ cohorts, alongside various other robust DMPs nominated from AD EWAS, would be of interest to the field. There is also the potential for cfDNA to be extracted from other biofluid types, such as CSF.

Interestingly, DNA methylation signatures have been used to develop "epigenetic clocks," which serve as biomarkers of aging. The first "epigenetic clock" was developed in 2013, using data generated across various tissues in many individuals across the lifespan, to predict chronological age [71], and since then many different clocks have been developed in the field. The purpose of epigenetic clocks is to predict the biological age of a tissue sample, which could in theory vary from the chronological age based on exposure environmental stresses and diseases. Indeed, studies have reported an association between accelerated epigenetic age in the cortex and neuropathological hallmarks of $\mathrm{AD}$ [72]. However, a recent clock that was trained specifically using cortical samples from older individuals did not observe any association of accelerated epigenetic age with neuropathology [73]. Therefore, the use of epigenetic clocks as a predictor of agerelated disease and longevity is still highly debated.

\section{The Future of Epigenomic Studies in Dementia}

There is now a growing field of literature reporting epigenomic alterations in dementia brain samples. Of note, many of the same loci have been identified across different studies, and pathway analyses consistently highlight an enrichment of differentially methylated loci in neuronal-related processes. Given that some loci have been consistently reported across different studies, tissue-specific meta-analyses should be undertaken on these datasets to identify robust changes in disease, particularly as some studies have been undertaken on relatively small sample sizes. However, there are some issues that require careful consideration when meta-analyzing epigenomic data. There are several sources of intra-individual variation that can confound data, for example different population backgrounds, collection of samples, and processing of data. The optimal way of minimizing the impact of patient-specific variation is through the collection of detailed information from individuals, such as demographic and lifestyle information, detailed clinical records, and the presence of co-morbidities, which can be incorporated as co-variates during the data analysis process. As discussed earlier in this review, mixed dementia is common and attempts should be made in both meta-analyses of existing data and future epigenomic studies to control for the presence of different neuropathological hallmarks, or to profile "pure" dementia cases, although this would considerably limit the study size. Although most of the dementia EWAS to date have been undertaken on cortical tissue, some of these have used different, distinct anatomical regions, with some studies even profiling multiple brain regions from the same donor and so consideration for this must be given during metaanalyses. In this vein, it is known that there are alterations in the abundance of different brain cell types in dementia in a brain region-specific manner and thus a larger magnitude of epigenetic changes in one brain region could simply reflect more gliosis or neurodegeneration present. There are methods that can be used to bioinformatically control for cell proportions [71, 74], which should be used during statistical analyses. Two recent studies have profiled neuronal and/or glia-specific DNA methylomes in dementia; however, these have been limited to just one or two cell populations and in a small number of samples. In the future, it would be of interest to isolate various cell proportions (e.g., different neuronal cell types, microglia, and astrocytes) and individually profile these, as well as undertaking analyses at the single-cell level.

Outside of the CNS, several EWAS have been undertaken exploring DNA methylation changes in dementia blood samples with the aim of identifying novel biomarkers for disease. Not only is it a relatively easy process to extract whole blood from a patient, but it can also provide live snapshots of the disease by allowing longitudinal assessments of individuals from before the onset of disease until death. However, what is evident from a handful of studies that have compared DNA methylation patterns between the brain and blood is that epigenomic signatures in the blood are distinct from the brain $[54,55]$, and that intraindividual variation that is mirrored across these tissues is largely driven by methylation quantitative trait loci (mQTL) [75]. Nonetheless, an accurate biomarker does not have to mirror the changes in the brain and could simply represent a specific peripheral response to alterations in the brain. However, it is known that there are subtle alterations in the abundance of different blood cell types in $\mathrm{AD} \mathrm{[76]} \mathrm{and} \mathrm{therefore} \mathrm{it} \mathrm{is} \mathrm{important} \mathrm{to} \mathrm{control}$ for these bioinformatically in analyses, as well as exploring epigenetic profiles in different cell populations. The potential to identify brain cell-specific DNA methylation signatures in circulating cfDNA is also an interesting avenue that should be pursued, particularly given these will be largely distinct from whole blood DNA methylation patterns. 
Although the majority of AD brain EWAS to date have been undertaken using the $450 \mathrm{~K}$ array, future studies will likely capitalize on technologies that profile more loci; the EPIC array allows quantification of over $850,000 \mathrm{CpGs}$, nearly twice the coverage of the $450 \mathrm{~K}$ array, while the falling cost of sequencing means that this also represents a promising new avenue. RRBS allows the enrichment of CG rich regions, giving better coverage of the genome than array-based methods, without redundant sequencing of regions that do not have $\mathrm{CpG}$ sites, which is one drawback with wholegenome bisulfite sequencing (WGBS). Another point of interest for $\mathrm{AD}$ would be single-cell methylomic sequencing, which would allow the identification of variation within cell types in disease. Although the focus of most studies has been on DNA methylation, bisulfite-based methods only give a summative measurement of DNA methylation and hydroxymethylation. A handful of studies have now profiled 5-mC and 5-hmC in isolation; however, these have been limited to low coverage of the genome (by using the $450 \mathrm{~K}$ array or low sequencing resolution) or a very small number of samples. Looking to the future, it will be of interest to profile a range of different DNA modifications, including both adenines and cytosines outside of a $\mathrm{CpG}$ context. Theoretically this could be facilitated using third-generation sequencing technologies such as the Oxford Nanopore Technologies (ONT) and Pacific Biosciences (Pacbio) platforms. These can allow the identification of DNA modifications on native DNA, although the current high cost and error rate mean these are not yet optimal for studying dementia. Nonetheless, continual developments in these technologies and the methods to subsequently analyze the data mean these could be a viable option in years to come. Although there appears to be a growing number of empirical research articles focussing on profiling DNA modifications in $\mathrm{AD}$, and more recently other dementias, there are relatively few studies profiling histone modifications. Of the two studies undertaken to date, both have used ChIP-seq to profile either H3K9ac or H3K27ac, which are both marks of active gene transcription. However, there are numerous other post-translational modifications that can occur to different histone proteins with varying effects on gene expression. Studies profiling a number of these marks in the same samples should be prioritized, perhaps using techniques such as Cleavage Under Targets and Release Using Nuclease (CUT\&RUN), which are reported to outperform ChIP-seq [77]. In addition, other techniques to probe chromatin accessibility, such as Assay for Transposase-Accessible Chromatin with high-throughput sequencing (ATAC-seq), should be undertaken in dementia samples.

Ultimately, the generation of large, comprehensive datasets of different epigenomic data modalities will allow a comprehensive insight into the epigenetic landscape in dementia. The integration of these datasets, with genomic, transcriptomic, and proteomic information, could ultimately allow the identification of key modulators that could represent novel therapeutic targets. One issue with identifying disease-associated epigenetic differences is that it is not known if these are driving the disease process or are secondary to pathology. However, recent advances in genome editing technology mean that it is now feasible to add or remove $5-\mathrm{mC}$ at specific sites in the genome [78]. Therefore, utilizing this tool for altering 5-mC in human cell lines and exploring the effect this has on cell function and pathological marks could allow us to establish whether epigenetic modifications are a cause or a consequence of developing dementia.

\section{Conclusions}

Epigenetics is a rapidly growing research area, particularly in the dementia field. Epigenomic studies have the potential to both further our understanding of the molecular etiology of disease as well as identify new therapeutic targets. Although previous studies have had a number of drawbacks, including issues around limited coverage, low sample size, sample heterogeneity, and inter-individual variation, the fact that many DNA methylomic studies in AD have identified the same loci suggests that there are robust alterations in disease. Looking to the future, researchers should aim to meta-analyze existing datasets as well as undertake new studies profiling large sample cohorts with detailed clinical and neuropathological data available. Such large-scale studies would allow well-powered analyses of "pure" dementia cases, as well as complex analyses of mixed dementia, which would give the most accurate interpretation of the complex epigenome within the dementia brain.

\section{Compliance with Ethical Standards}

Conflict of Interest There are no relevant disclosures for any of the authors.

Human and Animal Rights and Informed Consent This article does not contain any studies with human or animal subjects performed by any of the authors.

Open Access This article is licensed under a Creative Commons Attribution 4.0 International License, which permits use, sharing, adaptation, distribution and reproduction in any medium or format, as long as you give appropriate credit to the original author(s) and the source, provide a link to the Creative Commons licence, and indicate if changes were made. The images or other third party material in this article are included in the article's Creative Commons licence, unless indicated otherwise in a credit line to the material. If material is not included in the article's Creative Commons licence and your intended use is not permitted by statutory regulation or exceeds the permitted use, you will need to obtain permission directly from the copyright holder. To view a copy of this licence, visit http://creativecommons.org/licenses/by/4.0/. 


\section{References}

Papers of particular interest, published recently, have been highlighted as:

- Of importance

- Of major importance

1. Small G, Jarvik L. The dementia syndrome. 1982;320(8313):1443-6.

2. Prince M, Albanese E, Guerchet M, Prina M. World Alzheimer Report 2014: dementia and risk reduction an analysis of protective and modifiable factors. 2014.

3. Lane CA, Hardy J, Schott JM. Alzheimer's disease. Eur J Neurol. 2018;25(1):59-70

4. Mirra SS, Heyman A, McKeel D, Sumi S, Crain BJ, Brownlee L, et al. The Consortium to Establish a Registry for Alzheimer's Disease (CERAD): Part II. Standardization of the neuropathologic assessment of Alzheimer's disease. Neurology. 1991;41(4):479.

5. Braak H, Braak E. Neuropathological stageing of Alzheimerrelated changes. Acta Neuropathol. 1991;82(4):239-59.

6. Thal DR, Rüb U, Orantes M, Braak H. Phases of A $\beta$-deposition in the human brain and its relevance for the development of AD. Neurology. 2002;58(12):1791-800.

7. Paradise MB, Sachdev PS. Vascular cognitive disorder. Semin Neurol. 2019;39(2):241-50.

8. Kane JP, Surendranathan A, Bentley A, Barker SA, Taylor J-P, Thomas AJ, et al. Clinical prevalence of Lewy body dementia. Alzheimers Res Ther. 2018;10(1):19.

9. Hogan DB, Jetté N, Fiest KM, Roberts JI, Pearson D, Smith EE, et al. The prevalence and incidence of frontotemporal dementia: a systematic review. Can J Neurol Sci. 2016;43(S1):S96-S109.

10. McColgan P, Tabrizi SJ. Huntington's disease: a clinical review. Eur J Neurol. 2018;25(1):24-34.

11. Walker FO. Huntington's disease. Lancet. 2007;369(9557):218-28.

12. Peavy GM, Jacobson MW, Goldstein JL, Hamilton JM, Kane A, Gamst AC, et al. Cognitive and functional decline in Huntington's disease: dementia criteria revisited. Mov Disord. 2010;25(9):1163-9.

13. Cummings JL. Dementia with lewy bodies: molecular pathogenesis and implications for classification. J Geriatr Psychiatry Neurol. 2004;17(3):112-9.

14. Revesz T, McLaughlin JL, Rossor MN, Lantos PL. Pathology of familial Alzheimer's disease with Lewy bodies. J Neural Transm Suppl. 1997;51:121-35.

15. Schneider JA, Arvanitakis Z, Leurgans SE, Bennett DA. The neuropathology of probable Alzheimer disease and mild cognitive impairment. Ann Neurol. 2009;66(2):200-8.

16. Bateman RJ, Aisen PS, De Strooper B, Fox NC, Lemere CA, Ringman JM, et al. Autosomal-dominant Alzheimer's disease: a review and proposal for the prevention of Alzheimer's disease. Alzheimers Res Ther. 2011;3(1):1.

17. Zusso M, Barbierato M, Facci L, Skaper SD, Giusti P. Neuroepigenetics and Alzheimer's disease: an update. J Alzheimers Dis. 2018;64(3):671-88.

18. Tilley L, Morgan K, Kalsheker N. Genetic risk factors in Alzheimer's disease. Mol Pathol. 1998;51(6):293-304.

19.• Kunkle BW, Grenier-Boley B, Sims R, Bis JC, Damotte V, Naj $\mathrm{AC}$, et al. Genetic meta-analysis of diagnosed Alzheimer's disease identifies new risk loci and implicates $\mathrm{A} \beta$, tau, immunity and lipid processing. Nat Genet. 2019;51(3):414-30 This study represented the largest meta-analysis of GWAS in clinically diagnosed AD (94,437 individuals). The study reported 20 previous risk loci and five new genome-wide significant loci, two of which were also reported by Jansen et al. (2019). Pathway analysis implicates immunity, lipid metabolism, tau-binding proteins, and amyloid precursor protein (APP) metabolism.
20.• Jansen IE, Savage JE, Watanabe K, Bryois J, Williams DM, Steinberg S, et al. Genome-wide meta-analysis identifies new loci and functional pathways influencing Alzheimer's disease risk. Nat Genet. 2019;51(3):404-13 This represented the largest metaanalysis of GWAS in AD by leveraging on data from individuals "AD-by-proxy" based on parental diagnoses as well as clinically diagnosed cases. In total, 71,880 AD cases and 383, 378 controls were included, and 29 risk loci were identified, implicating 215 potential causative genes. The genes that were identified are strongly expressed in immune-related tissues and cell types and gene-set analyses nominated biological mechanisms involved in lipid-related processes and degradation of amyloid precursor proteins.

21. Karch CM, Goate AM. Alzheimer's disease risk genes and mechanisms of disease pathogenesis. Biol Psychiatry. 2015;77(1):43-51.

22. Escott-Price V, Sims R, Bannister C, Harold D, Vronskaya M, Majounie $\mathrm{E}$, et al. Common polygenic variation enhances risk prediction for Alzheimer's disease. Brain. 2015;138(Pt 12):3673-84.

23. Orme T, Guerreiro R, Bras J. The genetics of dementia with Lewy bodies: current understanding and future directions. Current Neurology and Neuroscience Reports. 2018;18(10).

24. Guerreiro R, Ross OA, Kun-Rodrigues C, Hernandez DG, Orme T, Eicher JD, et al. Investigating the genetic architecture of dementia with Lewy bodies: a two-stage genome-wide association study. Lancet Neurol. 2018;17(1):64-74.

25. Lu H, Liu X, Deng Y, Qing H. DNA methylation, a hand behind neurodegenerative diseases. Front Aging Neurosci. 2013;5:85.

26. Lev Maor G, Yearim A, Ast G. The alternative role of DNA methylation in splicing regulation. Trends Genet. 2015;31(5):274-80.

27. Richa R, Sinha RP. Hydroxymethylation of DNA: an epigenetic marker. EXCLI J. 2014;13:592.

28. Khare T, Pai S, Koncevicius K, Pal M, Kriukiene E, Liutkeviciute $\mathrm{Z}$, et al. 5-hmC in the brain is abundant in synaptic genes and shows differences at the exon-intron boundary. Nat Struct Mol Biol. 2012;19(10):1037-43.

29. Zhao S, Zhang X, Li H. Beyond histone acetylation - writing and erasing histone acylations. Curr Opin Struct Biol. 2018;53:169-77.

30. Salta E, De Strooper B. Noncoding RNAs in neurodegeneration. Nat Rev Neurosci. 2017;18(10):627-40.

31. Roubroeks JAY, Smith AR, Smith RG, Pishva E, Ibrahim Z, Sattlecker M, et al. An epigenome-wide association study of Alzheimer's disease blood highlights robust DNA hypermethylation in the HOXB6 gene. Neurobiol Aging. 2020. https://doi.org/ 10.1016/j.neurobiolaging.2020.06.023.

32. Barrachina M, Ferrer I. DNA methylation of alzheimer disease and tauopathy-related genes in postmortem brain. J Neuropathol Exp Neurol. 2009;68(8):880-91.

33. Brohede J, Rinde M, Winblad B, Graff C. A DNA methylation study of the amyloid precursor protein gene in several brain regions from patients with familial Alzheimer disease. J Neurogenet. 2010;24(4):179-81.

34. Guo X, Wu X, Ren L, Liu G, Li L. Epigenetic mechanisms of amyloid- $\beta$ production in anisomycin-treated SH-SY5Y cells. Neuroscience. 2011;194:272-81.

35. Pietrzak M, Rempala G, Nelson PT, Zheng J-J, Hetman M. Epigenetic silencing of nucleolar rRNA genes in Alzheimer's disease. PLoS One. 2011;6(7):e22585.

36. Fuso A, Seminara L, Cavallaro RA, D'Anselmi F, Scarpa S. Sadenosylmethionine/homocysteine cycle alterations modify DNA methylation status with consequent deregulation of PS1 and BACE and beta-amyloid production. Mol Cell Neurosci. 2005;28(1):195-204.

37. Rao JS, Keleshian VL, Klein S, Rapoport SI. Epigenetic modifications in frontal cortex from Alzheimer's disease and bipolar disorder patients. Transl Psychiatry. 2012;2:e132. 
38. Mastroeni D, McKee A, Grover A, Rogers J, Coleman PD. Epigenetic differences in cortical neurons from a pair of monozygotic twins discordant for Alzheimer's disease. PLoS One. 2009;4(8):e6617.

39. Chouliaras L, Mastroeni D, Delvaux E, Grover A, Kenis G, Hof PR, et al. Consistent decrease in global DNA methylation and hydroxymethylation in the hippocampus of Alzheimer's disease patients. Neurobiol Aging. 2013;34(9):2091-9.

40. Condliffe D, Wong A, Troakes C, Proitsi P, Patel Y, Chouliaras L, et al. Cross-region reduction in 5-hydroxymethylcytosine in Alzheimer's disease brain. Neurobiol Aging. 2014;35(8):1850-4.

41. Vasanthakumar A, Davis JW, Idler K, Waring JF, Asque E, RileyGillis B, et al. Harnessing peripheral DNA methylation differences in the Alzheimer's disease neuroimaging initiative (ADNI) to reveal novel biomarkers of disease. Clin Epigenetics. 2020;12(1):84.

42. Altuna M, Urdánoz-Casado A, Sánchez-Ruiz de Gordoa J, Zelaya MV, Labarga A, Lepesant JMJ, et al. DNA methylation signature of human hippocampus in Alzheimer's disease is linked to neurogenesis. Clin Epigenetics. 2019;11(1):91.

43. Lardenoije R, Roubroeks JA, Pishva E, Leber M, Wagner H, Iatrou A, et al. Alzheimer's disease-associated (hydroxy) methylomic changes in the brain and blood. Clin Epigenetics. 2019;11(1):164.

44. Konki M, Malonzo M, Karlsson IK, Lindgren N, Ghimire B, Smolander J, et al. Peripheral blood DNA methylation differences in twin pairs discordant for Alzheimer's disease. Clin Epigenetics. 2019;11(1):130.

45. Smith AR, Smith RG, Burrage J, Troakes C, Al-Sarraj S, Kalaria $\mathrm{RN}$, et al. A cross-brain regions study of ANK1 DNA methylation in different neurodegenerative diseases. Neurobiol Aging. 2019;74: $70-6$.

46. Gasparoni G, Bultmann S, Lutsik P, Kraus TFJ, Sordon S, Vlcek J, et al. DNA methylation analysis on purified neurons and glia dissects age and Alzheimer's disease-specific changes in the human cortex. Epigenetics Chromatin. 2018;11(1).

47. Madrid A, Hogan KJ, Papale LA, Clark LR, Asthana S, Johnson $\mathrm{SC}$, et al. DNA hypomethylation in blood links B3GALT4 and ZADH2 to Alzheimer's disease. J Alzheimers Dis. 2018;66(3): 927-34.

48. Smith RG, Hannon E, De Jager PL, Chibnik L, Lott SJ, Condliffe $\mathrm{D}$, et al. Elevated DNA methylation across a 48 -kb region spanning the HOXA gene cluster is associated with Alzheimer's disease neuropathology. Alzheimers Dement. 2018;14(12):1580-8.

49. Ellison EM, Bradley-Whitman MA, Lovell MA. Single-base resolution mapping of 5-hydroxymethylcytosine modifications in hippocampus of Alzheimer's disease subjects. Journal of Molec Neurosci. 2017.

50. Zhao J, Zhu Y, Yang J, Li L, Wu H, De Jager PL, et al. A genomewide profiling of brain DNA hydroxymethylation in Alzheimer's disease. Alzheimers Dement. 2017;13(6):674-88 This was the first study quantifying 5 -hmC in dementia comparing 20 AD and 10 control prefrontal cortex samples, identifying plaque and tangle-associated DhMRs. Although they were unable to completely discriminate between 5-hmC and 5-mC due to low sequencing resolution, the study evidenced a role for hydroxymethylation in dementia.

51. Kobayashi N, Shinagawa S, Nagata T, Shimada K, Shibata N, Ohnuma T, et al. Development of biomarkers based on DNA methylation in the NCAPH2/LMF2 promoter region for diagnosis of Alzheimer's disease and amnesic mild cognitive impairment. PLoS One. 2016;11(1).

52. Watson CT, Roussos P, Garg P, Ho DJ, Azam N, Katsel PL, et al. Genome-wide DNA methylation profiling in the superior temporal gyrus reveals epigenetic signatures associated with Alzheimer's disease. Genome Med. 2016;8(1).

53.• De Jager PL, Srivastava G, Lunnon K, Burgess J, Schalkwyk LC, $\mathrm{Yu}$ L, et al. Alzheimer's disease: early alterations in brain DNA methylation at ANK1, BIN1, RHBDF2 and other loci. Nat Neurosci. 2014;17(9):1156-63 This study represented the largest single EWAS of DNA methylation in Alzheimer's disease to date. The authors used the $450 \mathrm{~K}$ arrays to profile DNA methylation in the prefrontal cortex of $>700$ individuals, identifying 71 CpG sites associated with AD pathology, of which 11 were replicated in an independent dataset.

54. Yu L, Chibnik LB, Yang J, McCabe C, Xu J, Schneider JA, et al. Methylation profiles in peripheral blood CD4+ lymphocytes versus brain: the relation to Alzheimer's disease pathology. Alzheimers Dement. 2016;12(9):942-51.

55.• Lunnon K, Smith R, Hannon E, De Jager PL, Srivastava G, Volta $\mathrm{M}$, et al. Methylomic profiling implicates cortical deregulation of ANK1 in Alzheimer's disease. Nat Neurosci. 2014;17(9):1164-70 This study used the $450 \mathrm{~K}$ array to profile DNA methylation in matched entorhinal cortex, superior temporal gyrus, prefrontal cortex, and cerebellum brain samples from $\sim 120$ individuals with varying degrees of $A D$ pathology. They primarily focussed their analyses on a cortex-specific DMR within the ANK1 gene, which they replicated in three independent datasets. The study also profiled DNA methylation in whole blood collected from 57 of the donors but observed no overlap in AD-associated DMPs in the brain and blood.

56. Conrad C, Andreadis A, Trojanowski JQ, Dickson DW, Kang D, Chen X, et al. Genetic evidence for the involvement of $\tau$ in progressive supranuclear palsy. Ann Neurol. 1997;41(2):277-81.

57. Bakulski KM, Dolinoy DC, Sartor MA, Paulson HL, Konen JR, Lieberman AP, et al. Genome-wide DNA methylation differences between late-onset Alzheimer's disease and cognitively normal controls in human frontal cortex. J Alzheimer's Dis. 2012;29(3): 571-88.

58. Chuang YH, Lu AT, Paul KC, Folle AD, Bronstein JM, Bordelon Y, et al. Longitudinal epigenome-wide methylation study of cognitive decline and motor progression in Parkinson's disease. J Park Dis. 2019;9(2):389-400.

59. Sanchez-Mut JV, Heyn H, Vidal E, Moran S, Sayols S, DelgadoMorales R, et al. Human DNA methylomes of neurodegenerative diseases show common epigenomic patterns. Transl Psychiatry. 2016;6(1):e718-e.

60. Li Y, Chen JA, Sears RL, Gao F, Klein ED, Karydas A, et al. An epigenetic signature in peripheral blood associated with the haplotype on $17 \mathrm{q} 21.31$, a risk factor for neurodegenerative tauopathy. PLoS Genet. 2014;10(3).

61. Masliah E, Dumaop W, Galasko D, Desplats P. Distinctive patterns of DNA methylation associated with Parkinson disease. Epigenetics. 2013;8(10):1030-8.

62. Fernandez AF, Assenov Y, Martin-Subero JI, Balint B, Siebert R, Taniguchi $\mathrm{H}$, et al. A DNA methylation fingerprint of 1628 human samples. Genome Res. 2012;22(2):407-19.

63.• Klein H-U, McCabe C, Gjoneska E, Sullivan SE, Kaskow BJ, Tang A, et al. Epigenome-wide study uncovers large-scale changes in histone acetylation driven by tau pathology in aging and Alzheimer's human brains. Nat Neurosci. 2019;22(1):37-46 This represented the largest EWAS of histone acetylation in $\mathrm{AD}$ to date. The authors used ChIP-seq to profile H3K9ac in 669 donors, identifying amyloid- and tau-associated differentially acetylated. They reported that 17-DMAG, identified from the CMAP database, could be used to protect against tauassociated chromatin reorganization.

64.• Marzi SJ, Leung SK, Ribarska T, Hannon E, Smith AR, Pishva E, et al. A histone acetylome-wide association study of Alzheimer's disease identifies disease-associated $\mathrm{H} 3 \mathrm{~K} 27 \mathrm{ac}$ differences in the entorhinal cortex. Nat Neurosci. 2018;21(11):1618-27 This represented the first EWAS in AD using ChIP-seq to profile a histone modification (H3K27ac), studying 24 AD and 23 control entorhinal cortex samples. The authors identified 4162 differentially 
acetylated peaks and interestingly, and many of these were located in genes implicated in AD pathology or AD genetic risk variants. AD-associated differentially acetylated peaks were enriched in processes related to neuropathology and neuronal activity.

65. Mano T, Nagata K, Nonaka T, Tarutani A, Imamura T, Hashimoto $\mathrm{T}$, et al. Neuron-specific methylome analysis reveals epigenetic regulation and tau-related dysfunction of BRCA1 in Alzheimer's disease. Proc Natl Acad Sci. 2017;114(45):E9645-E54.

66. Smith AR, Smith RG, Pishva E, Hannon E, Roubroeks JAY, Burrage J, et al. Parallel profiling of DNA methylation and hydroxymethylation highlights neuropathology-associated epigenetic variation in Alzheimer's disease. Clin Epigenetics. 2019;11(1):52.

67. Fransquet PD, Lacaze P, Saffery R, McNeil J, Woods R, Ryan J. Blood DNA methylation as a potential biomarker of dementia: a systematic review. Alzheimers Dement. 2018;14(1):81-103.

68. Moss J, Magenheim J, Neiman D, Zemmour H, Loyfer N, Korach A, et al. Comprehensive human cell-type methylation atlas reveals origins of circulating cell-free DNA in health and disease. Nat Commun. 2018;9(1):1-12.

69. Mendioroz M, Martínez-Merino L, Blanco-Luquin I, Urdánoz A, Roldán M, Jericó I. Liquid biopsy: a new source of candidate biomarkers in amyotrophic lateral sclerosis. Ann Clin Transl Neurol. 2018;5(6):763-8.

70. Smith R, Pishva E, Shireby G, Smith AR, Roubroeks JA, Hannon $\mathrm{E}$, et al. Meta-analysis of epigenome-wide association studies in Alzheime's disease highlights novel differentially methylated loci across cortex. BioRxiv. 2020. https://doi.org/10.1101/2020.02.28. 957894
71. Horvath S. DNA methylation age of human tissues and cell types. Genome Biol. 2013;14(10):3156.

72. Levine ME, Lu AT, Bennett DA, Horvath S. Epigenetic age of the pre-frontal cortex is associated with neuritic plaques, amyloid load, and Alzheimer's disease related cognitive functioning. Aging. 2015;7(12):1198-211.

73. Shireby G, Davies J, Francis P, Burrage J, Walker E, Neilson G, et al. Recalibrating the epigenetic clock: implications for assessing biological age in the human cortex. bioRxiv. 2020.

74. Guintivano J, Aryee MJ, Kaminsky ZA. A cell epigenotype specific model for the correction of brain cellular heterogeneity bias and its application to age, brain region and major depression. Epigenetics. 2013;8(3):290-302.

75. Hannon E, Lunnon K, Schalkwyk L, Mill J. Interindividual methylomic variation across blood, cortex, and cerebellum: implications for epigenetic studies of neurological and neuropsychiatric phenotypes. Epigenetics. 2015;10(11):1024-32.

76. Lunnon K, Ibrahim Z, Proitsi P, Lourdusamy A, Newhouse S, Sattlecker M, et al. Mitochondrial dysfunction and immune activation are detectable in early Alzheimer's disease blood. J Alzheimers Dis. 2012;30(3):685-710.

77. Marinov GK. A decade of ChIP-seq. Oxford University Press; 2018.

78. Imm J, Kerrigan TL, Jeffries A, Lunnon K. Using induced pluripotent stem cells to explore genetic and epigenetic variation associated with Alzheimer's disease. Epigenomics. 2017;9(11):1455-68.

Publisher's Note Springer Nature remains neutral with regard to jurisdictional claims in published maps and institutional affiliations. 